\title{
Multicriteria Decision Analysis in Material Design, Selection, and Manufacturing
}

\author{
Ali Jahan, ${ }^{1}$ Kevin L. Edwards, ${ }^{2}$ Abbas S. Milani, ${ }^{3}$ and Marjan Bahraminasab ${ }^{4}$ \\ ${ }^{1}$ Department of Industrial and Mechanical Engineering, Islamic Azad University, Semnan Branch, Semnan, Iran \\ ${ }^{2}$ Institution of Engineering Designers, Courtleigh, Westbury Leigh, Wiltshire BE13 3TA, UK \\ ${ }^{3}$ School of Engineering, University of British Columbia, Kelowna, Canada V1V 1V7 \\ ${ }^{4}$ Department of Materials Science and Engineering, Buein Zahra Technical University, Buein Zahra, Qazvin, Iran
}

Correspondence should be addressed to Ali Jahan; a.jahan@semnaniau.ac.ir

Received 17 May 2015; Accepted 18 May 2015

Copyright (C) 2015 Ali Jahan et al. This is an open access article distributed under the Creative Commons Attribution License, which permits unrestricted use, distribution, and reproduction in any medium, provided the original work is properly cited.

The selection of a material for a specific engineering application necessitates that many criteria are satisfied at the same time. This can be a lengthy and expensive process, and often the final selection is based on compromise between advantages and disadvantages of candidate materials. Recently, multicriteria decision analysis has demonstrated significant capability and improvements in tackling complex engineering design problems. It has also begun to find applications in the optimization of advanced classes of materials, as well as material tailoring for a specific design, in addition to conventional materials selection problems. The main objective of this Special Issue is to provide a forum for discussing state-of-theart and novel ideas for producing added-value engineering products by concentrating on multicriteria decision analysis in material design, selection, and manufacturing. This Special Issue presents a judicious collection of recent research papers on both methodological and application aspects of strategic materials selection.

In the paper entitled "Intergranular Corrosion of Low Cr Ferritic Stainless Steel 429 Evaluated by the Optimized Double Loop Electrochemical Potentiokinetic Reactivation Test," the degree of sensitization to intergranular corrosion of FSS 429, which contains the interstitials of $0.017 \mathrm{wt} . \%$ $\mathrm{C}$ and $0.0048 \mathrm{wt} . \% \mathrm{~N}$ and the stabilizers of $0.12 \mathrm{wt} . \% \mathrm{Ti}$ and $0.32 \mathrm{wt} . \% \mathrm{Nb}$, is investigated using double loop electrochemical potentiokinetic reactivation test. The objective is to determine the optimum conditions (including electrolyte concentration, scan rate, and temperature) leading to a quantitative evaluation of the IGC susceptibility of FSS 429; then the specimens with different heat treatment have been tested in order to plot the time-temperature-sensitization curve of FSS 429. The paper entitled "A Fuzzy DelphiAnalytical Hierarchy Process Approach for Ranking of Effective Material Selection Criteria" is mainly a theoretical analysis in improving the decision-making processes for materials selection problems. An example is presented to demonstrate the proposed method. In the paper entitled "The Effects of Various Additive Components on the Sound Absorption Performances of Polyurethane Foams," flexible polyurethane foams with varying amounts of additive components are synthesized to improve sound damping and absorption. A two-microphone impedance tube has been applied to measure the sound absorption and transmission loss. In the paper entitled "Structure Optimization and Numerical Simulation of Nozzle for High Pressure Water Jetting," three kinds of nozzles normally used in industrial production are numerically simulated, and the structure of nozzle with the best jetting performance out of the three nozzles is optimized. In the paper entitled "Multicriteria Decision Analysis in Improving Quality of Design in Femoral Component of Knee Prostheses: Influence of Interface Geometry and Material," quality tools including QFD and DOE combined with FEA and the multicriteria decision analysis method are used for simultaneous component geometry and materials selection. 
In the final ranking of alternatives, the use of FGM was rated higher than conventional $\mathrm{Co}-\mathrm{Cr}$ in the design of a new femoral component.

By bringing together these key research papers in a special issue, the Guest Editors hope to enrich the journal readers with respect to the latest observations of multicriteria decision analysis in material design, selection, and manufacturing.

\section{Acknowledgments}

The quality of the published papers owes to the authors' excellence in research as well as the constructive feedback and comments received from the anonymous reviewers. We would like to express our sincere gratitude to all them for their great support in making this Special Issue possible.

Ali Jahan

Kevin L. Edwards

Abbas S. Milani

Marjan Bahraminasab 

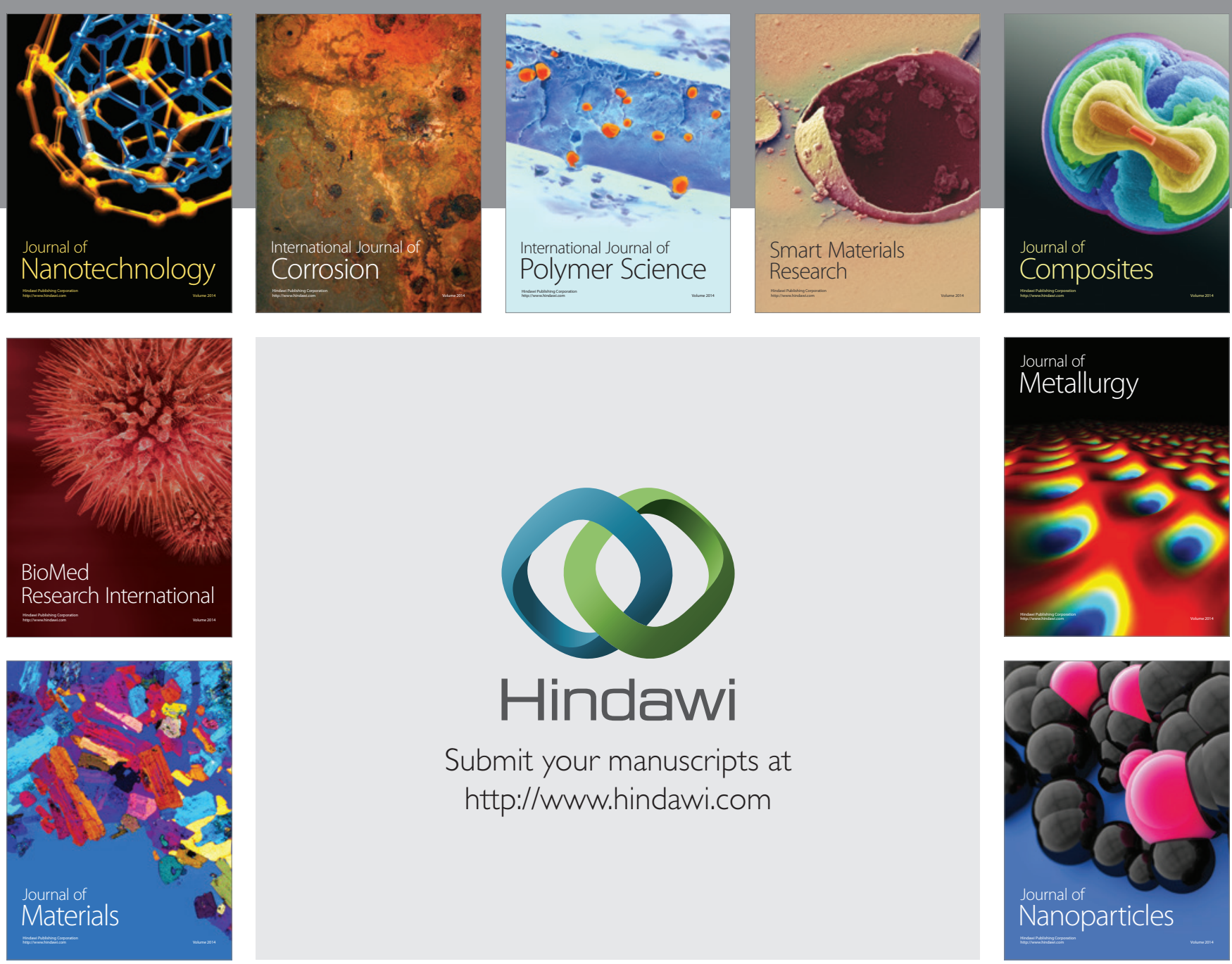

Submit your manuscripts at http://www.hindawi.com
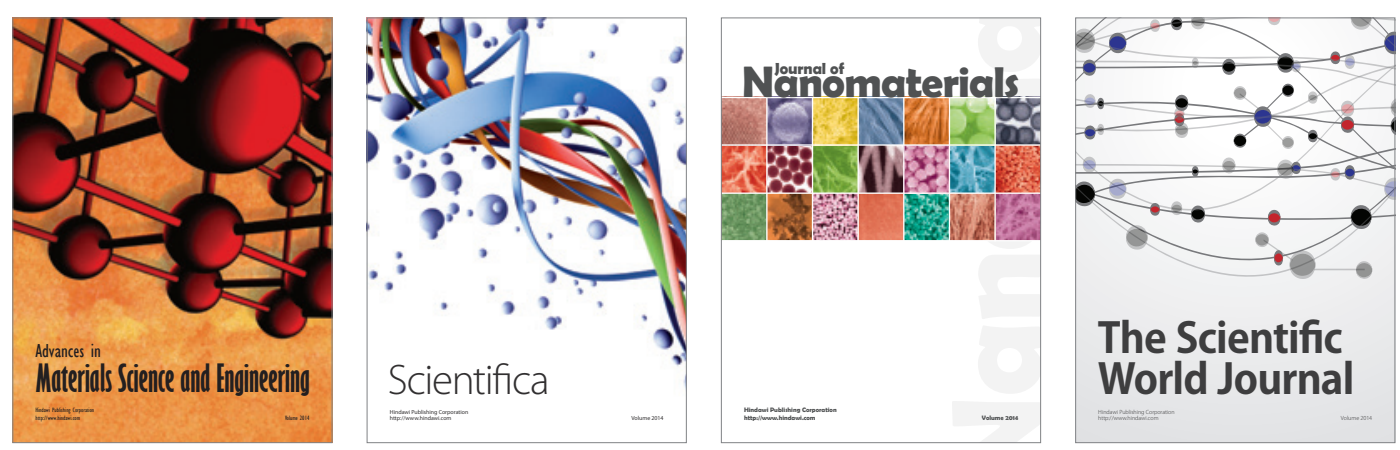

\section{The Scientific World Journal}
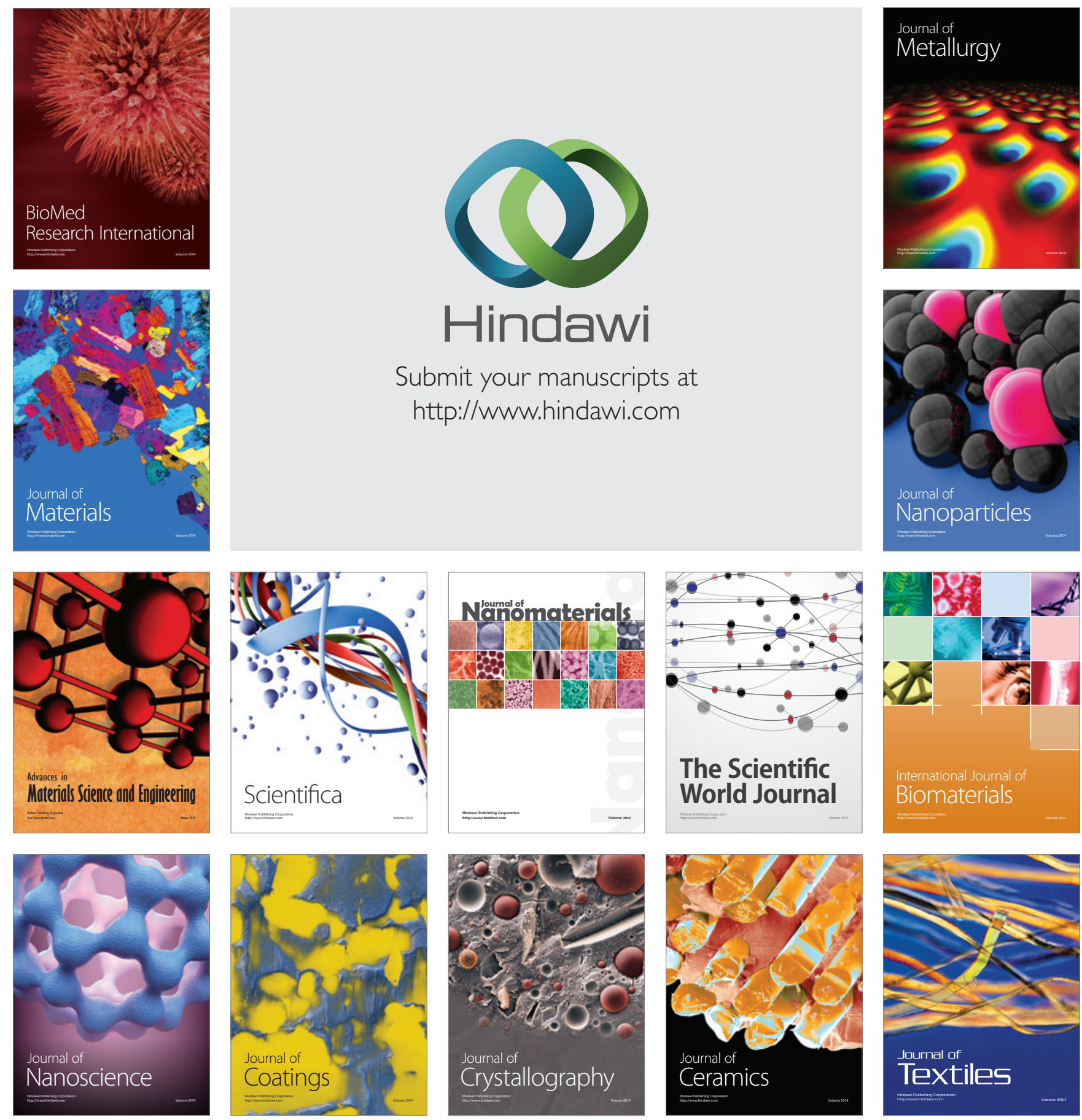